\title{
Editorial: Masonry arches, vaults and domes
}

\section{Gilbert PhD, CEng, MICE}

Masonry arches, vaults and domes have intrigued and fascinated researchers and practitioners for centuries, and continue to be of significant interest today. Such forms are found in historic buildings across the world and in bridges forming the backbone of the road and rail networks of many countries. It is very important that appropriate mechanical models are applied when these structures are assessed and when designing rehabilitation works.

This themed issue of Engineering and Computational Mechanics comprises ten contributions from leading international research workers and practitioners. Contributions cover a broad range of analytical and computational techniques, encompassing models based on plastic limit analysis principles, simplified dynamic analysis models and also elastic analysis models of varying levels of sophistication. The issue also contains an article focusing on masonry vault modal testing procedures.

In the first five contributions the powerful theorems of plastic limit analysis feature prominently. The first contribution is a briefing article by Harvey (2010), which highlights the potential for modern spreadsheets to be used to analyse complex three-dimensional masonry structures, making use of vector algebra in combination with the 'safe theorem' of plastic limit analysis. Practical guidance on setting up spreadsheets is provided, and the article should be of particular interest to practitioners needing to assess complex masonry structures.

The first full paper in this issue is authored by Jacques Heyman (Heyman, 2010), a pioneer of the application of plastic limit analysis principles to masonry structures. The author emphasises the difficulties which arise when conventional elastic analysis methods are applied, and stresses the benefits of applying traditional 'line of thrust' methods to verify the existence of a viable equilibrium state, thus confirming that the structure is safe. However, in his paper Heyman indicates that the well known 'safe theorem' of plastic limit analysis cannot, strictly speaking, be stated as 'the masonry must have sufficient thickness such that Hooke's inverted chain can be contained within the arch ring', and that in reality the orientation of the joints between voussoirs can have a small effect on arch capacity.

In their paper, Zessin et al. (2010) consider the stability of cracked masonry domes and present details of an iterative numerical procedure capable of evaluating the amount of support movement that can be sustained before collapse. The authors suggest that in a masonry dome the cracks which inevitably form when supports move outwards prevent tensile hoop stresses from being sustained, leading to increased forces being applied to the supports. The paper also documents results from parallel physical modelling studies, an interesting feature being the use of modern '3D printer' (or 'rapid prototyping') technology to fabricate masonry blocks of the correct geometry to form masonry dome test pieces.

Andreu et al. (2010) also consider the stability of threedimensional masonry forms, here using computer-simulated cable nets to confirm the existence of viable load paths. The proposed approach leads to highly visual models which are easy to interpret, and the approach can perhaps be seen as the natural successor to the three-dimensional physical hanging cable models used by Antoni Gaudi.

In the paper by Gilbert et al. (2010) a recently developed analysis procedure, discontinuity layout optimisation (DLO), is extended to allow rotational as well as translational failure mechanisms to be modelled. This means that DLO can be used to obtain rigorous upper bound limit-analysis solutions for arbitrary soil-masonry interaction problems, including masonry arch bridge problems involving soil backfill material. The authors, however, note that 'mobilised' rather than peak soil strengths need to be used in the analysis in order to obtain good correlation with experimental results.

The next two papers are concerned with analysis of the dynamic response of masonry arches, treating planar voussoir arches formed from rigid masonry blocks. The paper by Sinopoli (2010) describes in detail a semi-analytical approach to model the dynamics of a voussoir arch, designed to be used in conjunction with a suitable event-driven or time-stepping numerical procedure. DeJong and Ochsendorf (2010) describe results from a model involving a simplified, three-block, representation of an arch subject to in-plane seismic loading. Despite the simplified representation, the authors indicate that the model developed is conservative (i.e. failure is predicted for smaller earthquake amplitudes than observed experimentally) and also that, in reality, the ground accelerations caused by earthquakes can contain multiple consecutive impulses which are capable of having an amplifying effect, making accurate prediction of structural response somewhat difficult. 
In the paper by Wang and Melbourne (2010), attention turns to classical elastic analysis. A full derivation of the twopinned elastic formulation for masonry arch bridges developed by Pippard in the first half of the last century is presented and consequences of the various assumptions made are discussed. Given that Pippard's formulation underpins the MEXE method of assessment for masonry arch bridges still in relatively widespread usage today, outcomes from this study are potentially of significant practical interest. For example, the authors point out that one of the simplifications made by Pippard could be one of the reasons why the MEXE method sometimes over-estimates the load carrying capacity of short span bridges.

In the paper by Brookes (2010) application of an altogether more powerful 'elastic' analysis procedure is considered, with the author describing the use of a mixed finite/discrete-element modelling approach to assess masonry arch bridges before and after strengthening works. Brookes points out that whilst conventional (implicit) finite-element analysis approaches can suffer from serious numerical difficulties when modelling masonry structures, such difficulties can be avoided through the use of the mixed finite/discrete-element (explicit) modelling approach described. The approach can provide information both prior to, and at, the collapse limit state, and has to date been applied to over 200 bridges.

The final paper by Atamturktur et al. (2010) considers the relative merits of two experimental modal analysis (EMA) methods for application to masonry vaults. The information derived from EMA tests is potentially useful for validating numerical analysis models and for use in structural health monitoring studies (where the usual goal is to relate changes in vibration response to changes in the structural state of a building). The authors conclude that the operational modal testing approach, in which church bells or an orchestra might typically be used as an excitation source, can provide a viable alternative to traditional approaches, involving excitation via a hammer or shaker.

The wide range of analytical and computational techniques covered in this themed issue, by authors from around the world, is indicative of the strong interest in the area, and it seems likely that masonry arches, vaults and domes will continue to intrigue and fascinate researchers and practitioners alike for many years to come!

\section{REFERENCES}

Andreu A, Gil L and Roca P (2010) Analysis of masonry structures by funicular networks. Proceedings of the Institution of Civil Engineers, Engineering and Computational Mechanics 163(3): 147-154, doi: 10.1680/eacm.2010. 163.3.147.

Atamturktur S, Fanning P and Boothby TE (2010) Traditional and operational modal testing of masonry vaults. Proceedings of the Institution of Civil Engineers, Engineering and Computational Mechanics 163(3): 213-223, doi: 10.1680/eacm.2010.163.3.213.

Brookes C (2010) Application of the finite/discrete element method to arches. Proceedings of the Institution of Civil Engineers, Engineering and Computational Mechanics 163(3): 203-211, doi: 10.1680/eacm.2010.163.3.203.

DeJong MJ and Ochsendorf JA (2010) Dynamics of in-plane arch rocking: an energy approach. Proceedings of the Institution of Civil Engineers, Engineering and Computational Mechanics 163(3): 179-186, doi: 10.1680/eacm.2010. 163.3.179.

Gilbert M, Smith CC and Pritchard TJ (2010) Masonry arch analysis using discontinuity layout optimisation. Proceedings of the Institution of Civil Engineers, Engineering and Computational Mechanics 163(3): 155-166, doi: 10.1680/ eacm.2010.163.3.155.

Harvey W (2010) Briefing: Spreadsheet analysis of complex masonry structures. Proceedings of the Institution of Civil Engineers, Engineering and Computational Mechanics 163(3): 125-128, doi: 10.1680/eacm.2010.163.3.125. Heyman J (2010) Equilibrium of masonry arches. Proceedings of the Institution of Civil Engineers, Engineering and Computational Mechanics 163(3): 129-133, doi: 10.1680/ eacm.2010.163.3.129.

Sinopoli A (2010) A semi-analytical approach for the dynamics of the stone arch. Proceedings of the Institution of Civil Engineers, Engineering and Computational Mechanics 163(3): 167-178, doi: 10.1680/eacm.2010.163.3.167. Wang J and Melbourne C (2010) Mechanics of MEXE method for masonry arch bridge assessment. Proceedings of the Institution of Civil Engineers, Engineering and Computational Mechanics 163(3): 187-202, doi: 10.1680/eacm.2010. 163.3.187.

Zessin J, Lau W and Ochsendorf J (2010) Equilibrium of cracked masonry domes. Proceedings of the Institution of Civil Engineers, Engineering and Computational Mechanics 163(3): 135-145, doi: 10.1680/eacm.2010.163.3.135. 